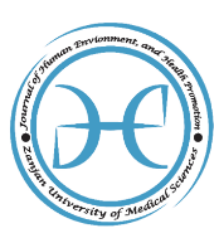

Journal of Human, Environment and Health Promotion

Journal homepage: www.zums.ac.ir/jhehp

\title{
The Effect of Rotator Cuff Muscles Exercises on Range of Motion, Proprioception and Electrical Activity in Male Volleyball Players with Shoulder Joint Internal Rotation Deficit
}

\author{
Mohsen Moradi ${ }^{\text {a,* }}$ (D) | Malihe Hadadnechad ${ }^{a}$ | Amir Letafatkar ${ }^{a}$ \\ a Department of Corrective Exercise and Sport Injury, School of Physical Education and Sport Sciences, Kharazmi University, \\ Tehran, Iran.
}

${ }^{*}$ Corresponding author: Mohsen Moradi

Department of Corrective Exercise and Sport Injury, School of Physical Education and Sport Sciences, Kharazmi University, Tehran, Iran, 4515174999. Tell: +98-9193450353.

E-mail address: Mohsenmoradi90@gmail.com

\section{A R T I C L E I N F O}

Article type:

Original Article

\section{Article history:}

Received December 13, 2017

Revised January 28, 2018

Accepted February 17, 2018

DOI: $10.29252 /$ jhehp.3.2.72

Keywords:

Specific Exercises

Rotator Cuff Muscles

Proprioception

Electrical Activity

Shoulder Joint Internal Rotation Defici

\begin{abstract}
A B S T R A C T
Background: The shoulder joint internal rotation deficit is inter alia the most important risk factors for throwing athletes. Thus, the present study aims at investigating the effect of eight weeks of rotator cuff muscles' specific exercises on the range of motion, proprioception and the activation time of the selective shoulder muscles in male volleyball players with shoulder joint internal rotation deficit.

Methods: Thirty male athletes with shoulder joint internal rotation deficit took part in the present study and after preliminary screening and identification of the qualified athletes, the testes were assigned to two groups: experimental $(n=15)$ and control $(n=$ 15).

Results: The results of the statistical tests indicated that rotator cuff muscles' specific exercises have had a significant effect on the range of motion, proprioception and electrical activation of the anterior deltoid muscle, medial deltoid muscle, posterior deltoid muscle, infraspinatus and supraspinatus.

Conclusion: According to the positive influence of the rotator cuff muscles' specific exercises, it is recommended that these rotator cuff muscles' specific exercises should be added to the rehabilitation-therapy programs for male volleyball players with shoulder joint internal rotation deficit so that the limitations resulting from such a deficit in the range of motion could be eased.
\end{abstract}

\section{Introduction}

Overhead throwing move is a complex activity necessitating a good deal of skilfulness and it exerts too much pressure on the shoulder joint system [1]. The performance stability of the shoulder is the result of the mutual relationship between the static and dynamic stabilizers and such a relationship comes about via the sensory-motor (proprioceptive) system.

Proprioception is the perfection of the touching sense incorporating the movement and position senses [2,3].
The proprioceptive receptors are located on skin, muscles, joints as well as the ligaments and tendons and they can transform the mechanical deformations to neural signals.Nowadays, proprioception is an integral part of sport-related injuries rehabilitation programs [4]. Joint lesions can influence the proprioception and disrupt the natural neuromuscular reactions served to protect the joints $[5,6]$. Internal rotation deficit is the range of motion differential of the dominant and non-dominant glenohumeral joint internal rotations that adversely influences the biomechanics of the glenohumeral joint during throwing and overhead throwing activities as performed in volleyball [7]. 
Therefore, the internal rotation range of motion deficit is considered as a risk factor for throwing athletes and overhead throwing athletes like the volleyball players [7]. It is reported in several studies that the glenohumeral internal rotation is degraded more in the throwing shoulder than the opposite shoulder and, covnersely, the glenohumeral external rotation is strengthened in the former $[7,8]$.

Such inconsistent changes in the throwing athletes' shoulders are called glenohumeral internal rotation range of motion deficit [7]. The glenohumeral internal rotation deficit is in the first place associated with soft tissue problems like the muscular tightness (posterior deltoid, infraspinatus and teres minor-muscles), flexibility, capsular tightness and muscular weakness $[9,10]$. The researchers have used electromyography to investigate the activities of shoulder muscles in the athletes and ordinary individuals when throwing. The researches showed that the rotator cuff play a protective role when throwing [11]. Resolving the internal rotation deficit is important in preventing shoulder injuries.

The treatments include stretching exercises to provide for more flecibility of the dorsal glenohumeral muscles [12,13], muscle movability for the treatment of the joint capsule tightness and the other forms of manual treatments $[14,15]$.

The internal rotationd deficit can cause imbalance in the musclular endurance, shoulder internal impingement syndrome, ligamentous laxity, tightness of the joint's posterior capsule, tightness and shortness of the posterior shoulder muscles, reduction of the glenohumeral internal rotation, the reduction of scapula's range of motion and lack of coordination and balance between the shoulder and scapular region muscles [12-15]. The use of screening methods and evaluation of these elements and reduction and moderation of them can reduce the occurrence likelihood of the injury. The use of physical exercise as a cheap, accessible, noninvasive and less-risky means of maintaining healthiness and dynamicity and preservation or recovery of balance has been recommended as an acceptable tool for the reduction of pain and enhancement of the range of motion [16]. The majority of the studies have employed stretching exercises for treating the individuals with glenohumeral internal rotation deficit.

According to the fact that the internal rotation deficit, besides bringing about tightening of the posterior shoulder elements, can influence the muscular strength, range of motion and proprioception of the shoulder joint, the present study is thus seeking to find an answer to the question as to whether eight weeks of rotator cuff muscle specific exercises is effective on the range of motion, proprioception and electrical activity initiation time of the selected shoulder muscles of the male volleyball players with glenohumeral internal rotation deficit or not.

\section{Materials and Methods}

The present study population is consisted of the academic male volleyball players with the glenohumeral internal rotation deficit. Out of the whole study population, 30 individuals were selected as the study sample volume according to the study inclusion criteria based on a purposive method and using Gpower software. The study sample volume was next assigned to experimental and control groups, each containing 15 subjects. The testees whose differential of the internal rotaton in both of the dominant and nondominant shoulders was 18 degrees were diagnosed as the individuals with glenohumeral internal rotationd deficit. Also, any past history of fracture and surgery in the shoulder joint and other disorders influencing the study course and the lack of regular presence in the exercise programs for three sessions a week were considered as the study exclusion criteria. The individuals with glenohumeral internal rotation deficit were diagnosed using goniometer. In a continuation stage of the pretest, proprioception was evaluated using isokinetic device. To measure the initiation time of the muscles' electrical activity using electromyography, the subjects were asked to perform shoulder elevation (abduction) assignment. Next, the exercise group members were subjected to eight weeks of totator cuff specific exercises for 45 to 60 minutes, three sessions a week; these exercises were comprised of stretching exercises, strength exercises (resistence training by the use of a red theraband), neuromuscular and functional exercises. Before the commencement of the exercises protocol by the intervention group, the study subjects were asked to perform warmup for ten minutes that included easy running and performing stretching moves and also to perform cool-down exercises for five minutes at the end of each session. In the end of the week eight, the posttest was started under the same conditions of the pretest and, finally, the results were analyzed.

\subsection{Measuring the Glenohumeral Internal Rotation Range of Motion}

To evaluate the range of inward rotation while the subject was asked to lie down on a bed in a supine position (Figure 1), it was explained to the testes that they have to keep their shoulder girdle muscles in a completely relaxed situation. The shoulder was placed in a 90-degree abduction on the edge of the bed and the elbow was also formed a 90-degree angle with the bed. The examiner exerted an amount of force by one hand on the subject's forearm to move his shoulder joint passively about the coronal axis inward and his other hand was placed on the acromioclavicular joint. Then, the internal rotation was ceased as soon as movement was felt in the acromioclavicular joint and the subject's hand was kept fixed by anohter person. This way, the internal rotation range of motion of the shoulder about the coronal axis was calculated. To increase the accuracy of the measurement and reduce the test error, the glenohumeral external rotation range of motion was evaluated three times and the mean value of the three evaluations was scored. The entire stages were repeated for the dominant and nondominant shoulder. The differential of the range of motion showing a value above 18 degrees in the internal rotation for dominant and nondominant organs was considered as range of motion deficit of the volleyball players [17]. 


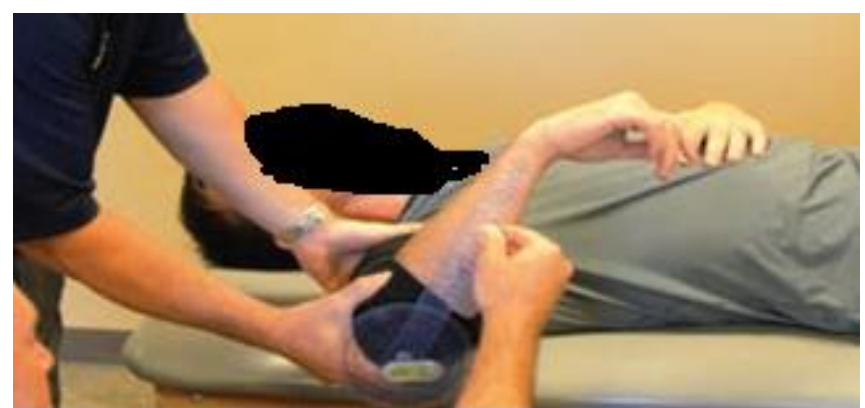

Figure 1: Measuring the glenohumeral internal rotation range of motion

\subsection{Measuring the Electrical Activity Initiation Time Using Electromyography}

In electromyography examinations, only the surficial scoring of the supraspinatus and infraspinatus muscles is possible inter alia the rotator cuff muscles. Scoring the electrical activity of the subscapularis and tres-minor muscles can only be done by using wire electrodes. Also, the electrical activities of the posterior deltoid, medial deltoid and anterior deltoid were scored. The electrode was installed based on Soniyam method. To score the electrical activity of the infraspinatus muscle, the electrode was placed on a part of the more superficial muscle, i.e. on the upper trapezius muscle's wing that is less voluminous. So, the electrode was placed in the exterior one third of the muscle aligned with its fibers' direction. To compute scores from deltoid muscle in its anterior part, the electrode was placed within a $1-\mathrm{cm}$ distance from the coracoid process, for its middle section, the active electrode was placed within a distance between acromion and deltoid knob and for the posterior section, the electrode was placed beneath the posterior glenoid surface [18]. The amounts of the muscles' activities during internal rotation of the shoulder starting from a neutral position (Figure 2). The electromyographical information was collected by the use of an electromyography device (MIE model, featurng 8 channels and a $1000-\mathrm{Hz}$ sampling frequency, made in England). The signals were preamplified ten times stronger and subjected to a band-pass filtering between $20 \mathrm{~Hz}$ and $500 \mathrm{~Hz}$. The obtained values from mean square root calculations were divided by the values of the maximum voluntary contraction of each muscle so as to compare the results between the study subjects and also to normalize the extracted data and the muscles' electrical activities were offered as percentages of the maximum voluntary contraction of the muscles. Each situation of maximum voluntary contraction was repeated twice for a period of five seconds and then the mean value of the middle three-second of maximum voluntary contraction was utilized for normalization of the data [19].

To calculate the muscles' activities initiation times, at first the unidirectional waves, three times the standard deviation values of the muscles' electrical activities, were recognized as the baseline activity initiation threshold. Conventionally, the activity initiation time was set at the point where the muscle activity hit the threshold and remained at least 25 milliseconds above its level. Electromyography signals were passed in a program written by electronic engineer in MATLAB environment through $50-\mathrm{Hz}$ notch filter and then the cases mentioned above in regard of the signals were taken into account for the recognition of the muscle activity [18].

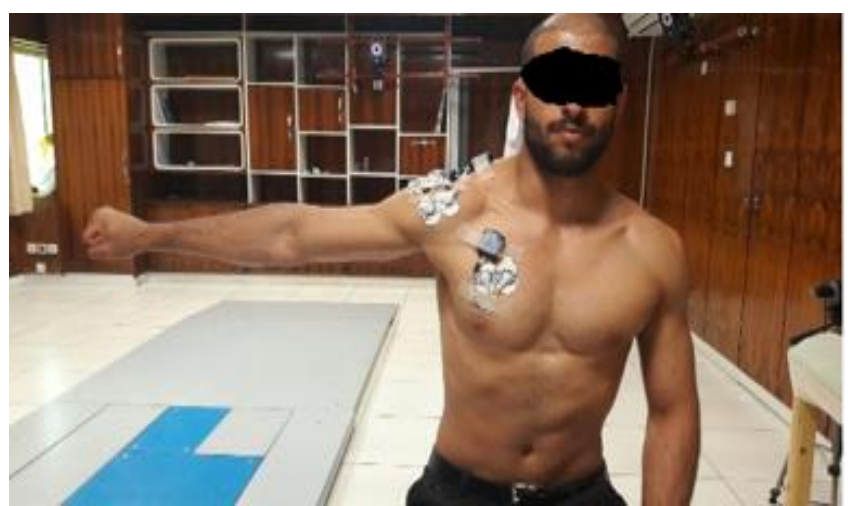

Figure 2: Measuring the electrical activity initiation time using electromyography

\subsection{Evaluation of the Rotator Cuff Muscles' Proprioception Using Isokinetic System}

To measure the shoulder position proprioception, there was made use of an isokinetic device, Biodex System Model made in the US. To do so, at first the subjects were made familiar with the device and the test method was explained to them. The study subjects were blindfolded and asked to sit at the Isokinetic dynamometer chair. The straps were fastened on the study subjects' chests so as to refrain any additional moves of their upper extremity. The launching position for a 90-degree angle of the chair back was $45^{\circ}$ and it was $90^{\circ}$ for the 45-degree chair back angle in the rehabilitation phase. The study subject's shoulder was actively positioned in each of the three target angles and kept there for ten seconds. The subject was asked to concentrate on the angle and then to return his arm actively to the starting position. Then the subject was asked to take a 5second break. The process was repeated twice and the study subject was asked to for a third time rotate his arm actively so as to hit the target angle. When it was felt by the study subject that he has reached the target angle, he was asked to cease the lever arm from moving. The process was repeated thrice and the differential modulus of the recorded angle and the target angle was scored as the error value (absolute angular error) and finally the mean values were computed to undergo further analysis [20].

The mean and standard deviation of the study subjects' demographic characteristics have been summarized in table 1 .

Table 1: Subjects' demographic characteristics

\begin{tabular}{llll}
\hline Group & $\begin{array}{l}\text { Age (years) } \\
\text { Mean } \pm \text { Sd }\end{array}$ & $\begin{array}{l}\text { Weight }(\mathbf{k g}) \\
\text { Mean } \pm \text { Sd }\end{array}$ & $\begin{array}{l}\text { Height }(\mathbf{c m}) \\
\text { Mean } \pm \text { Sd }\end{array}$ \\
\hline $\begin{array}{l}\text { Internal rotation } \\
\text { deficit }\end{array}$ & $23.87 \pm 4.43$ & $74.93 \pm 2.62$ & $179.84 \pm 6.52$ \\
Control & $23.43 \pm 3.79$ & $73.46 \pm 15.6$ & $181.29 \pm 6.93$ \\
\hline
\end{tabular}


According to the data normality, table 2 presents the information pertaining to the descriptive pretest and posttest statistics of the groups in terms of the studied variables. As it is observed, the mean values of the internal rotator muscles' internal rotation and the proprioception have undergone an increase following the exercises and that the mean value of the muscles' electrical activity initiation has decreased and also that the mean values obtained for the control group regarding the aforementioned variables have remained unchanged in the course of the study time.

The results of the covariance analyses for the group comparisons (table 3 ) indicates that there is a significant difference between the groups suggesting the effect of the independent variable on the dependent variable, i.e. the rotator cuff specific exercises have caused an improvement in the proprioception, reduction of the electrical activity initiation of the muscles and increase in the extent of the shoulder joint's internal rotation.

\section{Results and Discussion}

The results of the present study indicated that the rotator cuff muscles' specific exercises have a significant effect on the proprioception, timing of the medial deltoid, supraspinatus and infraspinatus muscles as well as the shoulder joint's range of motion in such a way that the proprioception was found improved and the timing of the medial deltoid, supraspinatus and infraspinatus muscles were found decreased following eight weeks of prescribed exercises; but, it was also Figured out that the rotator cuff specific exercises have not significant effects on the anterior deltoid and posterior deltoid muscles' activity initiation and it was documented that the range of shoulder joint's motions in the individuals with internal rotation deficit have undergone an increase.

These changes were not evidenced for the control group. In regard of the proprioception, the results of the present study are consistent with the findings of the studies by Foruhideh et al. (2010), Roy et al. (2009) and Moharrami et al. (2013) [19,21,22].

Also, the results of the current research paper in the foresaid regard do not conform to what has been found by Joe et al. (2011) in a research titled "evaluation of the effect of the internal rotator muscles' fatigue on the shoulder joint proprioception" reported that the internal rotator muscles' fatigue does not influence the shoulder and scapula proprioception [23].

Also, Foruhideh et al. (2011) investigated the short-term effect of exertion of vibration on the whole body on the shoulder joint proprioception in young and healthy study samples to show that these exercises cause an improvement in the shoulder joint proprioception even after a session of exercise [21].

Moharrami et al. (2015) investigated the effect of theraband exercises on the postural sense of the internal and external rotator muscles of the male athletes with shoulder impingement syndrome to demonstrate that these theraband exercises bring about a significant improvement in the postural senses of the both shoulder external and internal rotator muscles groups composed of male athletes with shoulder impingement syndrome [19]. Roy et al. (2009), in a research named "the effect of resistance training and movement control exercises on the shoulder performance of the individual with shoulder impingement syndrome,

Table 2: The information pertaining to the descriptive pretest and posttest statistics of the groups in terms of the studied

\begin{tabular}{|c|c|c|c|c|}
\hline \multirow[t]{2}{*}{ Variable } & & \multirow[t]{2}{*}{ Group } & \multirow{2}{*}{$\begin{array}{c}\text { Pretest } \\
\begin{array}{c}\text { Mean and standard } \\
\text { deviation }\end{array}\end{array}$} & \multirow{2}{*}{$\begin{array}{c}\text { Posttest } \\
\begin{array}{c}\text { Mean and standaro } \\
\text { deviation }\end{array}\end{array}$} \\
\hline & & & & \\
\hline \multirow{2}{*}{\multicolumn{2}{|c|}{ The amount of shoulder's internal rotation }} & Internal rotation deficit & $28.73 \pm 5.17$ & $39.60 \pm 5.48$ \\
\hline & & Control & $29.13 \pm 4.70$ & $28.53 \pm 4.73$ \\
\hline \multirow{2}{*}{\multicolumn{2}{|c|}{ Proprioception }} & Internal rotation deficit & $5.45 \pm 2.34$ & $3.33 \pm 2.15$ \\
\hline & & Control & $4.93 \pm 2.17$ & $5.20 \pm 2.26$ \\
\hline \multirow[t]{10}{*}{ Activity initiation time } & Anterior deltoid & Internal rotation deficit & $437.73 \pm 25.38$ & $417.53 \pm 26.90$ \\
\hline & & Control & $433.06 \pm 23.20$ & $431.80 \pm 24.94$ \\
\hline & Medial deltoid & Internal rotation deficit & $424.13 \pm 30.90$ & $397.52 \pm 34.89$ \\
\hline & & Control & $421.80 \pm 30.87$ & $422.46 \pm 29.66$ \\
\hline & Posterior deltoid & Internal rotation deficit & $502.60 \pm 25.60$ & $514.13 \pm 22.49$ \\
\hline & & Control & $504.46 \pm 25.45$ & $503.26 \pm 24.25$ \\
\hline & supraspinatus & Internal rotation deficit & $485.13 \pm 35.35$ & $435.93 \pm 20.95$ \\
\hline & & Control & $480.86 \pm 33.67$ & $483.66 \pm 32.69$ \\
\hline & Infraspinatus & Internal rotation deficit & $477.01 \pm 27.52$ & $424.13 \pm 28.87$ \\
\hline & & Control & $469.53 \pm 30.66$ & $475.89 \pm 28.81$ \\
\hline
\end{tabular}


Table 3: The results of the covariance analyses for the group Comparisons

\begin{tabular}{lcc} 
Table 3: The results of the covariance analyses for the group Comparisons & F & Root square of the mean \\
\hline Variable & $\boldsymbol{P}$ value & 26.521 \\
\hline Proprioception & 0.049 & 1568.192 \\
Anterior deltoid muscle's activity initiation time & 2.391 & 4706.079 \\
Medial deltoid muscle's activity initiation time & 4.389 & 8.134 \\
Posterior deltoid muscle's activity initiation time & 1.586 & 0.046 \\
Supraspinatus muscle's activity initiation time & 25.537 & 16847.553 \\
Infraspinatus deltoid muscle's activity initiation time & 24.875 & 19719.118 \\
The extent of the shoulder's internal rotation & 36.767 & 9.219 \\
\hline
\end{tabular}

concluded that the resistance training and movement control exercises cause an improvement in the shoulder performance [22]. One possible reason behind the inconsistency in the results is the exercise protocol employed to influence proprioception. The researchers have concluded that the rehabilitation exercises should incorporate muscular balance, muscular endurance, gradual improvement of the proprioception, dynamic persistence and neuromuscular control; the cuff rotator muscles' specific exercises encompass all these cases [2-10]. Cuff rotator muscles' specific exercises lead to the neuromuscular training which can per se improve the proprioception [24]. Exercise is usually accompanied by learning. Perceptual learning means enhancing the capacity of sensory distinction following a series of exercises. Perceptual learning can result in the improvement of signal processing in a familiarized situation thereby to increase the precision of the postural sense. According to the interventions made through training with cuff rotator specific exercises and that the proprioception is more dependent on the receptors extant on the muscle and the joint, the muscles' receptors play more accentuated roles with the effect of the exercises on the muscles, particularly during performing active moves. Upon the muscles being stretched during the movement cycles, the rate of the muscular spindles' stimulation becomes more than the state that the muscles are in their unstretched and short positions and this is closely associated with the precision of the joint's postural sense and awareness of the status of the body's joints. Also, the simultaneous activity of the gamma nerves during the active contraction of the muscles occasionally leads to the increase in the ascending activity of the muscle spindles and the muscles that are activated at the same time elevate the proprioceptive precision through augmenting the sensitivity to stretch the activated muscle spindles in the periphery of the joint. Finally, it seems that the main reason why the proprioception is strengthened is the stimulation of the proprioceptive receptors during the closed-chain neuromuscular exercises.

The results of the present study regarding the muscles' timing is in compliance with the researches performed by Matin et al. (2010) and Zolnour et al. [25, 26]; but, they were found inconsistent with the results obtained by Cools et al. (2003), De May et al. (2012) and Lucas et al. (2000) [11, 27,28]. Matin et al. (2010) investigated the muscular activity pattern of the shoulder girdle of the women in some common physiotrapical exercises of the upper extremity to express that the behavior of the upper trapezius muscle is not correlated with the developmental motor pattern form, whereas the behavior pattern of the pectoralis major muscle was found associated with the developmental motor pattern.

The activity of the anterior and posterior deltoid muscle depends on the direction in which the motor pattern is developed and the activity of the medial deltoid muscle depends on the target joint's displacement in a developmental motor pattern; also, the activities of the medial trapezius muscle and infraspinatus muscle, as the stabilizer-guider muscles, do not depend on the muscular activity patterns [25].

Zolnour et al. (2018) in a research entitled "the timing and the intensity of the shoulder muscles in handball throwing movements in the individuals with shoulder impingement syndrome and healthy individuals" reported that the intensity rates of the upper and dorsal major trapezius muscles' activities are 50.74 and 42.43 higher in the healthy group than the patient group, respectively. But, the intensity of the medial deltoid muscle's activity in the healthy individuals was $38.50 \%$ smaller than the patients. In healthy individuals, the supraspinatus muscle was activated 23.5 milliseconds earlier than performing the throwing move and the triceps was activated about 18.5 milliseconds later than performing the throwing move [26]. In line with these results, Cools et al. (2003) found no significant difference between the group with the shoulder pain syndrome and the healthy group in terms of the activation time of the upper trapezius muscle [11]. De May et al. (2012) investigated the effect of six weeks of rehabilitation exercises on the timing and performance of the shoulder muscles in individuals with shoulder pain syndrome to show that there is no significant difference in regard of the muscle activation time before and after taking the exercises [27].

Amongst the possible reasons behind the inconsistency of the results, the differences in the testes, exercise duration and exercise type can be pointed out. The activities of the anterior and posterior deltoid muscles seem to predominantly be in line with movement neutralization of their counteracting counterparts. In the meanwhile, in some movement patterns, especially the ones carried out in an inclined manner such as the movement pattern of performing abduction with internal rotation, the behaviors of these two muscles depend on the direction at which the movement pattern is performed. Therefore, the timing of the posterior and anterior deltoid muscles' timing was not found varied as a result of the cuff rotator specific exercises. Delay in the activation of the muscles stabilizing and/or controlling the movements of the upper extremity caused by shoulder defect can facilitate the occurrence of damage. Parallel to this, 
Janda, as well, realizes delay in muscle activation as being caused by muscular weakness and believes that the delay in the muscle activity initiation provides for the expression of incorrect or abnormal movement pattern. Thus, offering cuff rotator specific exercises causes correct movement pattern through reducing the activation time of such muscles as supraspinatus, infraspinatus and anterior deltoid as a result of which the damage likelihood of the shoulder injury goes down. Also, disruption in the neuromuscular performance of the muscles in the periphery of the shoulder as observed in the athletes with glenohumeral internal rotation deficit can be attributed to the change in predesigned programs sent from the central nervous system to the muscles. Any change in the predesigned programs sent from the central nervous system as was documented herein can be justified as put forth in the following statement: the movement restriction as manifested in the internal rotation of the shoulder results in disordering of the shoulder proprioception in the long-term.

Disorder in the shoulder proprioception per se leads to the change in the afferent messages issued by the nervous system. The varied afferent messages can cause neurophysiological changes, including the change in the motor commands issued to the muscles. Also, due to its comprehensiveness and inclusion of the majority of the exercise characteristics seen in the majority of the shoulder functional activities, the exercise program used in the present study possesses potentials that can strengthen the majority of the motor function aspects including the proprioception. These programs can possibly send more complete information via the sensory receptors to the brain in which case a greater many of the neurons can be influences if the relationship between the several receptors and the brain is established. In fact, corresponding to the principles of synapsis and myelin production, utilization of the stimulations stemming from the neuromuscular exercises along with an array of the other training measures that can facilitate movement actualization seems to result in the creation of more synapses in the cerebral cortex hence more myelination results that can eventually provide for a better control of the movement. Therefore, with their effect on the shoulder proprioception, the rotator cuff muscles' specific exercises cause changes in the afferent messages issued by the nervous system and such a change in the afferent messages in itself bring about changes in the motor commands issued to the muscles as a result of which the activity initiation times of the supraspinatus, infraspinatus and anterior deltoid muscles will be eventually reduced.

In regard of the range of motion, the results of the present study are in accordance with the results obtained in the studies by Aldridge et al. (2012), Cools et al. (2012) and Oyama et al. (2010) [4, 29, 10]. Also, the present study results were found inconsistent with the findings of 30 . Jurgel et al. (2005) [30]. Aldridge et al. (2012) reported that daily stretching exercises cause an increase in the internal rotation range of motion as well as in the total range of motion [4]. Cools et al. (2012) subjected the posterior shoulder structure to stretching exercises and reported that the stretching exercises bring about improvement in the internal rotation deficit [29].

Hall et al. (2012), as well, concluded that all three types of the active, passive and Proprioception Neuromuscular Facilitation (PNF) stretching exercises cause an increase in the shoulder's internal rotation range of motion and there was found no significant differences between these three types of exercises [15]. In line with this, several researchers reported that PNF exercises, performed in one repetition, cause an increase in the range of motion and that the range of motion variations are scored in a range from $3^{\circ}$ to $9^{\circ}$ and depend on the joint $[6,12]$. Jurgel et al. (2005) exposed the individuals with frozen shoulder and average age of over 50 years, to four weeks of rehabilitation exercises to Figure out a significant effect on pain reduction, strength increase and improvement of the range of motion in shoulder joint when flexing, extending, abducting and adducting. But, no significant effect of the exercises was documented in internal and external arm rotation moves [30]. Inter alia the possible reasons behind such an inconsistency of the results, one can point to the differences in the exercise duration, age, exercise protocol and the disorder type. The rehabilitation exercises' mechanism (combined sport exercises) for the shoulder is in such a way that they provide for the reacquiring of the natural range of motion and mitigation of pain in the shoulder joint through enhancing the blood circulation and nourishing the shoulder joint, prevention of atrophy development and relaxation of the shoulder muscles as well as creation of a sense of comfort and self-confidence in the patient and relieving of pain in the damaged tissues $[14,31]$.

The individuals with internal rotation deficit are faced with pain, limitation and reduction of range of motion in the shoulder joint in their performing of the active and passive moves and the disease is treated and the shoulder restores its natural state in case that the pain and range of motion are improved. Therefore, the imposed exercise programs as described herein aimed at increasing the stretchability of the soft tissues, reduction of the posterior-lower shoulder capsule and increase in the flexibility so as to enhance the shoulder joint's internal rotation range of motion. Moreover, the rotator cuff muscles' specific exercises have possibly strengthened the shoulder muscle, increased the muscle strength and reduced the drynesses and adhesions inside the joint so that the reduction in the movement restriction and enhancement of the range of motion could be brought about.

\section{Conclusion}

According to the results obtained in the current research paper, it seems that the rotator cuff muscle's specific exercises cause an improvement in the proprioception and range of motion in the individuals with shoulder joint's internal rotation deficit. Furthermore, these exercises decrease the shoulder muscles' activation time. Thus, the performing of these exercises is suggested for the individuals with the foresaid deficit in line with resolving the deficit and prevention of the subsequent damages. 


\section{Authors' Contributions}

M.M., M.H., and A.L., designed the study and wrote the manuscript; M.M., conducted the experimental work; M.M., M.H., and A.L., analyzed the data. All authors revised and approved the final manuscript.

\section{Conflict of Interest}

The authors affirm that there is no conflicts of interest that may have influenced the preparation of this manuscript.

\section{Acknowledgments}

We thank all volleyball players Kharazmi University who assisted in subject preparation and data collection.

\section{References}

1. Wilk KE, Meister K, Andrews JR. Current Concepts in the Rehabilitation of the Overhead Throwing Athlete. Am J Sports Med. 2002; 30(1):136-51.

2. Ellenbecker TS. Shoulder Rehabilitation. Non-Operative Treatment. New York: Thieme, 2006:180.

3. Riemann BL, Lephart SM. The Sensorimotor System, Part I: The Physiologic Basis of Functional Joint Stability. J Athl Train. 2002; 37(1): 71 .

4. Aldridge R, Guffey JS, Whitehead MT, Head P. The Effects of a Daily Stretching Protocol on Passive Glenohumeral Internal Rotation in Overhead Throwing Collegiate Athletes. Int J Sports Phys Ther. 2012; 7(4): 365.

5. Bahr R, Bahr IA. Incidence of Acute Volleyball Injuries: A Prospective Cohort Study of Injury Mechanisms and Risk Factors. Scand J Med Sci Sports. 1997; 7(3): 166-71.

6. Borsa PA, Laudner KG, Sauers EL. Mobility and Stability Adaptations in the Shoulder of the Overhead Athlete: A Theoretical and Evidence-Based Perspective. Sports Med. 2008; 38(1): 17-36.

7. Kibler WB, Sciascia A, Thomas SJ. Glenohumeral Internal Rotation Deficit: Pathogenesis and Response to Acute Throwing. Sports Med Arthrosc Rev. 2012; 20(1): 34-8.

8. Kolt GS. Physical Therapies in Sport and Exercise. $2^{\text {nd }}$ ed. Elsevier Health Sciences; 2007.

9. Laudner KG, Sipes RC, Wilson JT. The Acute Effects of Sleeper Stretches on Shoulder Range of Motion. J Athl Train. 2008; 43(4): 359-63.

10. Oyama S, Goerger CP, Goerger BM, Lephart SM, Myers JB. Effects of Non-assisted Posterior Shoulder Stretches on Shoulder Range of Motion Among Collegiate Baseball Pitchers. Athl Ther Sport Health Care. 2010; 2(4): 163-70.

11. Cools AM, Witvrouw EE, Declercq GA, Danneels LA, Cambier DC. Scapular Muscle Recruitment Patterns: Trapezius Muscle Latency with and without Impingement Symptoms. Am J Sports Med. 2003; 32(4): 542-9.

12. Crockett HC, Gross LB, Wilk KE, Schwartz ML, Reed J, et al. Osseous Adaptation and Range of Motion at the Glenohumeral Joint in Professional Baseball Pitchers. Am J Sports Med. 2002; 30(1): 20-6.

13. Lucas KR, Polus BI, Rich PA. Temporal Sequence of Muscle Recruitment during Scapular Plane Elevation. Department of Chiropractic. 2000; 17(6): 341-8.

14. Salamh PA, Kolber MJ, Hanney WJ. Effect of Scapular Stabilization during Horizontal adduction stretching on Passive Internal Rotation and Posterior Shoulder Tightness in Young
Women Volleyball Athletes: A Randomized Controlled Trial. Arch Phys Med Rehabil. 2015; 96(2): 349-56.

15. Hall J,Oliver GD, Stone AJ. Comparison of Active, Passive, and Proprioceptive Neuromuscular Facilitation Stretching for Improving Glenohumeral Internal Rotation. Athl Ther Sports Health Care. 2012: 5(2): 1-7.

16. Lin YL, Karduna A. Four-week Exercise Program does not Change Rotator Cuff Muscle Activation and Scapular Kinematics in Healthy Subjects. J Orthop Res. 2016 1; 34(12): 2079-88.

17. Guney H, Harput G, Colakoglu F, Baltaci G. Glenohumeral Internal Rotation Deficit Affects Functional Rotator Strength Ratio in Adolescent Overhead Athletes. J Sport Rehabil. 2016; 25(1): 52-7.

18. Haddad Nejad. Comparison of the Effect of Functional and Palmometric Stability Exercises on Some of the Pulmonary Tonsil Electromyography Indices in Active Women with Trunk Control Defect (Susceptible to Anterior Cruciate Ligament Injury). Tehran: University of Tehran; 2013. [In Parsian].

19. Mohammadi R, Shojaeddin Sadreddin, Sadeghi H. The Effect of Trabant Exercises on the Sense of Position of Internal and External Muscles of the Shoulder of the Athlete's Men with Traumatic Syndrome. J Rehabil. 2013; 16(3). [In Parsian].

20. Senbursa G, Baltaci G, Atay A. Comparison of Conservative Treatment with and without Manual Physical Therapy for Patients with Shoulder Impingement Syndrome: A Prospective, Randomized Clinical Trial. Knee Surg Sports Traumatol Arthrosc. 2007; 15: 915-21.

21. Frohideh F, Kalantari Kh, Rahimi A, Farhadi A. The Study of the Short duration Effect of a Session on Total Body Vibration on the Isokinetic Strength of Rotator Cuff Muscles and Shoulder Sensation in Young and Healthy Samples. Sci J Kurdistan Univ Med Sci. 2010; 16: 24-6. [In Persian].

22. Roy JS, Moffet H, Hébert LJ, Lirette R. Effect of Motor Control and Strengthening Exercises on Shoulder Function in Persons with Impingement Syndrome: A Single-Subject Study Design. Man Ther. 2009; 14(2): 180-8.

23. Guo LY, Lin CF, Yang CH, Hou YY, Chen SK, Lan W. Evaluation of Internal Rotator Muscle Fatigue on Shoulder and Scapular Proprioception. J Mech Med Biol. 2011; 11(3): 663-74.

24. Pappas AM, Zawacki RM, Sullivan TJ. Biomechanics of Baseball Pitching: A Preliminary Report. Am J Sports Med. 1985; 13(4): 216-22.

25. Matin M, Ebrahimi A, Shaterzadeh M. Evaluation of the Belt Muscle Activity Pattern of Women in Some Common Exercises of Upper Limb Physicaltherapy. J Rehabil. 2010; 11(2): 49-58. [In Parsian].

26. Zolnour Z, Farahpour N, Jafarnejad Agro A. Timing and Intensity of Shoulder Muscle Activity in Handball Throwing Movement in Patients with Shoulder Syndrome and Healthy People. Res Rehabil Sci. 2017; 13 (1): 36-43. [In Parsian].

27. De May K, Danneels L, Cagnie B, Cools AM. Scapular Muscle Rehabilitation Exercises in Overhead Athletes with Impingement Symptoms: Effect of a 6-Week Training Program on Muscle Recruitment and Functional Outcome. Am J Sports Med. 2012; 40: 1906-15.

28. Lucas KR, Polus BI, Rich PA. Temporal Sequence of Muscle Recruitment during Scapular Plane Elevation. Department of Chiropractic. 2000; 17(6): 341-8.

29. Cools AM, Johansson FR, Cagnie B, Cambier DC, Witvrouw EE. Stretching the Posterior Shoulder Structures in Subjects with Internal Rotation Deficit: Comparison of Two Stretching Techniques. Shoulder Elbow. 2012; 4(1): 56-63.

30. Jurgel J, Rannama L, Gapeyeva H, Ereline J, Kolts I, Paasuke M. Shoulder Function in Patients with Frozen Shoulder before and after 4-Week Rehabilitation. Med (Kaunas, Lithuania). 2005; 41(1): 30-8.

31. Wells SN, Schilz J, Uhl TL, Gurney AB. A Literature Review of Studies Evaluating Rotator Cuff Activation during early Rehabilitation Exercises for Post-op Rotator Cuff Repair. Rehabil Sci Faculty Publications. 2016. 